\title{
Patterns of Connections in Rat Visual Cortex
}

\author{
Rafael Malach \\ Center for Neurosciences and Behavioural Research, The Weizmann Institute of Science, Rehovot, Israel 76100
}

The definition of visual areas is one of the central problems in visual cortex research. Rodent extrastriate cortex offers a striking example of the complexity of this issue, in that different parcelation schemes identify within it from 2 to as many as 13 separate visual areas. In the experiments reported here, patterns of connections within rat visual cortex were studied in an effort to better define its organizational layout.

The experimental paradigm used consisted of the following steps: first, the pattern of callosal connections was revealed in vivo with the fluorescent tracer bisbenzimide. Then, using the callosal pattern as a landmark, single injections of WGA-HRP were placed at various sites in striate and extrastriate cortex. Subsequently, the relation between the tangential distribution of ipsilateral corticocortical connections, the callosal connections, and the borders of striate cortex were examined in the flattened cortex preparation.

The experiments revealed widespread, patchy connections within rat visual cortex. These connections appeared to reflect 3 organizational trends. First, neighboring sites were more extensively connected than distant ones. Second, extrastriate sites receiving common striate cortex inputs tended to be interconnected. Finally, projections from opposite poles in striate cortex tended to form interdigitating patterns of connections in regions of overlap.

Altogether these trends suggest that the extrastriate band adjoining striate cortex has a single, global map organization. However, within the global map, a clear modular organization was evident, which appeared to correspond to the multiple visuotopic representations reported for this region. Based on its location, and some organizational similarities, it is suggested that the global map may constitute the rat homolog of area $v 2$ In cat and monkey.

One of the more intensely studied questions in cortical (particularly visual cortex) research is the issue of multiple cortical representations of the sensory surface. Why are there multiple cortical maps? A common notion is that such multiplicity might reflect cortical specialization, whereby various aspects of the visual information are processed separately by individual areas (e.g., Van Essen and Maunsell, 1983; Desimone et. al., 1985; Barlow, 1986).

Given the functional significance of this architecture, the issues of how to parcel the cortical tissue into individual areas

Received Dec. 22, 1989; revised Apr. 10, 1989; accepted May 12, 1989.

I wish to thank M. Harel and A. Rattner for technical assistance and T. Hendler and $Y$. Citri for helpful comments. This work was supported by grants from the Israel Institute for Psychobiology and the U.S.-Israel B.S.F. grant 85-00258.

Correspondence should be addressed to Rafael Malach at the above address.

Copyright (C) 1989 Society for Neuroscience $0270-6474 / 89 / 113741-12 \$ 02.00 / 0$ and what is the definition of a cortical area are of substantial interest. Several criteria for such definition, based on cytoarchitecture, electrophysiological mapping, and connectional organization, including relationship to callosal connections (Van Essen et al., 1982), have been proposed (for a comprehensive review of this issue, see Van Essen, 1985). However, the problem of cortical parcelation is difficult since in many instances cortical areas cannot be easily delineated and the different criteria do not converge on the same parcelation schemes.

In this respect, rat visual cortex provides a striking example of the problem. Originally, it was divided, on cytoarchitectonic grounds, into 3 areas: a primary area 17 (V1, striate cortex) and 2 extrastriate areas: area $18 \mathrm{a}$ on the lateral side and area $18 \mathrm{~b}$ on the medial side (Krieg, 1946; Caviness, 1975). Support for such a scheme came also from most electrophysiological mapping studies in other rodents such as the mouse (Drager, 1975; Wagor et al., 1980), golden hamster (Tiao and Blakemore, 1976), guinea pig (Choudhury, 1978), and gray squirrel (Hall et al., 1971).

At the other extreme, a more recent parcelation scheme based on connectional and physiological mapping experiments (Olavarria and Montero, 1984; Thomas and Espinoza, 1987) identified within the same territory as many as 13 separate visual areas, suggesting rat extrastriate cortex is a miniature version of cat and monkey cortices with their more than dozen currently defined areas.

The claim for high multiplicity of areas in rat extrastriate cortex is based on connectional studies showing that single tracer injections in central parts of area V1 result in multiple patches of projections (Olavarria and Montero, 1981). These patches are neatly localized inside callosal rings revealed in the flattened cortex preparation (Cusick and Lund, 1981; Olavarria and Montero, 1984). Furthermore, based on electrophysiological mapping experiments, it has been reported that extrastriate cortex contains multiple representations of the visual field (Montero et al., 1973a; Espinoza and Thomas, 1983; Thomas and Espinoza, 1987). While these results could suggest a true multiplicity of areas in extrastriate cortex, they are also compatible with a single map organization, albeit containing substantial visuotopic irregularities.

Recently we have developed an experimental approach which could assist in addressing the issue of extrastriate parcelation in the rat. The method, termed vital tract tracing (VTT), is based on visualizing the pattern of callosal connections in vivo using the fluorescent tracer bisbenzimide (BB) (Malach, 1987, 1988b). Taking advantage of the fixed relation between the callosal pattern and cortical subdivisions, we could achieve greater precision in targeting injections of a second tracer at chosen cortical sites.

Thus, the VTT approach made it feasible to study projections 


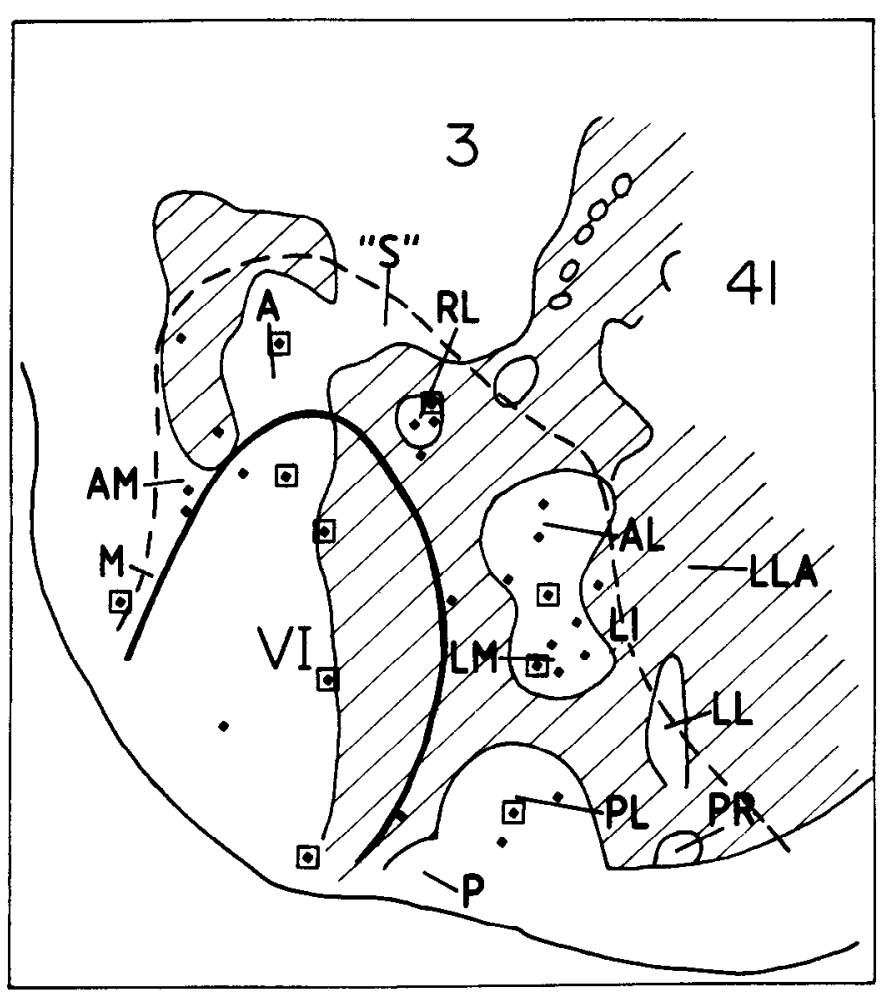

Figure 1. Overview of all the injection sites (diamonds) in this study, showing their relation to the borders of area 17 (VI, thick line), areas $18 \mathrm{a}$ and $18 \mathrm{~b}$ on the lateral and medial sides of area 17 , respectively (dashed line), the callosally connected regions (diagonal lines), and the extrastriate zones referred to in the text. These include, moving clockwise around area 17: the medial $(M)$, anteromedial $(A M)$, anterior $(A)$, "somatosensory" (" $S$ "), rostrolateral $(R L)$, anterolateral $(A L)$, lateromedial $(L M)$, laterointermediate $(L I)$, posterolateral $(P L)$, posterior $(P)$, laterolateral anterior $(L L a)$, laterolateral $(L L)$, and pararhinal $(P R)$ zones. Cases included in the figures are indicated by a square around their injection sites.

of the extreme poles of striate cortex as well as various zones, defined by callosal rings, within extrastriate cortex. Using this approach could reveal whether the entire striatal map is connected to each of the extrastriate subdivisions and whether instrinsic extrastriate connections are local, confined to neighboring sites, or extend throughout the entire extrastriate cortex. Such data could assist in clarifying the parcelation layout within rat visual cortex.

The results presented here suggest that the belt of tissue adjoining striate cortex has complex organization. On a global scale, this territory conforms to a single map organization, while locally, it is exceedingly fragmented and contains independent sets of distinct modules.

Some of the data have appeared previously in brief reports (Malach, 1987, 1988a).

\section{Materials and Methods}

This study contains data from 31 adult, hooded rats in which wheat germ agglutinin conjugated to HRP (WGA-HRP) was injected into various locations within striate and extrastriate cortex. In all cases except one, the callosal pattern was revealed in vivo and was used to assist in targeting the WGA-HRP injection sites. The details of the methods employed in this study and particularly the VTT approach, which is a novel application of the fluorescent tracing technique, have been described fully elsewhere (Malach, 1988b). Briefly, hooded rats were anesthetized with a mixture of ketamine (Vetalar, $100 \mathrm{mg} / \mathrm{kg}$ ) and xylazine (Rompun, $24 \mathrm{mg} / \mathrm{kg}$ ). A solution of $10 \%$ BB (Hoechst \#33258; Sigma) in distilled water was injected in 15-20 evenly distributed sites across the left posterior cortical hemisphere. Total volume injected was 4-5 $\mu \mathrm{l}$, tip of the injection pipette was $40-100 \mu \mathrm{m}$.

Following 4-6 days survival, the animals were reanesthetized, the right cortical hemisphere was exposed, soaked with $1 \mathrm{mg} / \mathrm{ml}$ epinephrine solution to constrict epidural blood vessels, and illuminated with 365 nm UV light to reveal the BB-labeled callosal pattern.

The fluorescing pattern was viewed through a low-power (5-25x) stereo microscope and occasionally photographed through a $500 \mathrm{~nm}$ long-pass filter. Using the callosal pattern as a landmark, it was fairly straightforward to place tracer injections consistently and precisely at chosen targets. Once the injection site was selected, a 20-60 $\mu \mathrm{m}$ tipped pipette filled with 5\% WGA-HRP was inserted into the cortex, and the tracer was iontophoretically injected for $15-45$ min using $5 \mu \mathrm{A}, 7 \mathrm{sec}$ on-7 sec off DC current (pipette positive). After $2 \mathrm{~d}$, the animals were deeply anesthetized with chloral hydrate and perfused with $0.1 \mathrm{M}$ PBS containing 10,000 units of heparin. This was followed by either $2 \%$ glutaraldehyde in PBS and 5\% sucrose or 4\% formaldehyde in PBS and $5 \%$ sucrose. The latter fixative gave somewhat weaker HRP staining but much lower fluorescence background.

In most cases, following perfusion, the cortical mantle was separated from the rest of the brain, flattened between 2 glass slides, and then sectioned tangentially to the surface at $40 \mu \mathrm{m}$ (Welker and Woolsey, 1974; Olavarria and Montero, 1984); the rest of the brain was cut coronally at $60 \mu \mathrm{m}$.

Flat sections were photographed unstained at low power using transmitted light illumination and high-contrast film (Kodak LPD-4) to reveal area 17 borders (see below) and then were either processed for HRP or incubated in $0.1 \%$ sodium nitroprusside solution to reduce BB fading. HRP reaction was a modified TMB method (Mesulam, 1978; Malach and Graybiel, 1986).

The sections were viewed using dark field and cross-polarization (Illing and Wassle, 1979) to reveal the WGA-HRP labeling pattern or epilluminated with UV light to reveal the BB-labeled patterns. Neighboring sections were aligned using vascularization patterns.

\section{Results}

To assist in referring to injection sites and locations of labeled patches, extrastriate cortex was subdivided into zones related to callosal rings and formations according to the detailed nomenclature proposed by Montero and coworkers (Montero et al., 1973b; Olavarria and Montero, 1984; Thomas and Espinoza, 1987). The issue whether these zones also correspond to individual visual areas will be dealt with in the discussion.

Figure 1 shows a summary diagram of all the sites injected in this study and their relation to the callosal related zones. Also shown are the myeloarchitectonically defined area 17 , area $18 \mathrm{a}$, and area $18 \mathrm{~b}$ borders.

The scheme adopted here divides extrastriate cortex into a belt of zones immediately abutting area 17, and an additional tier of zones situated more laterally, all related to callosal rings or bands. The subdivisions bordering area 17 are named, moving from caudal to rostral: zones posterior $(P)$, posterolateral $(\mathrm{PL})$, lateromedial (LM), and anterolateral (AL), all corresponding to a series of laterally located callosal rings situated in cytoarchitectonically defined area $18 \mathrm{a}$. More rostrally, zone rostrolateral (RL) is easily identified by its association with a ringlike formation rostral to the main callosal band called the anterior occipital callosal ring (AOCR), and anteromedial to it is a region bordering somatosensory cortex termed " $\mathrm{S}$." Moving into area $18 \mathrm{~b}$ on the medial side, one encounters zones anterior (A) and anteromedial (AM), which are separated by a commashaped callosal band. Most posteriorly on the medial side is zone medial (M). Besides these central subdivisions immediately adjoining area 17 , there are more laterally situated zones laterolateral anterior (LLa), laterolateral (LL), laterointermediate (LI), and most laterally, encroaching the rhinal fissure, zone pararhinal (PR). These zones were less accessible for study 

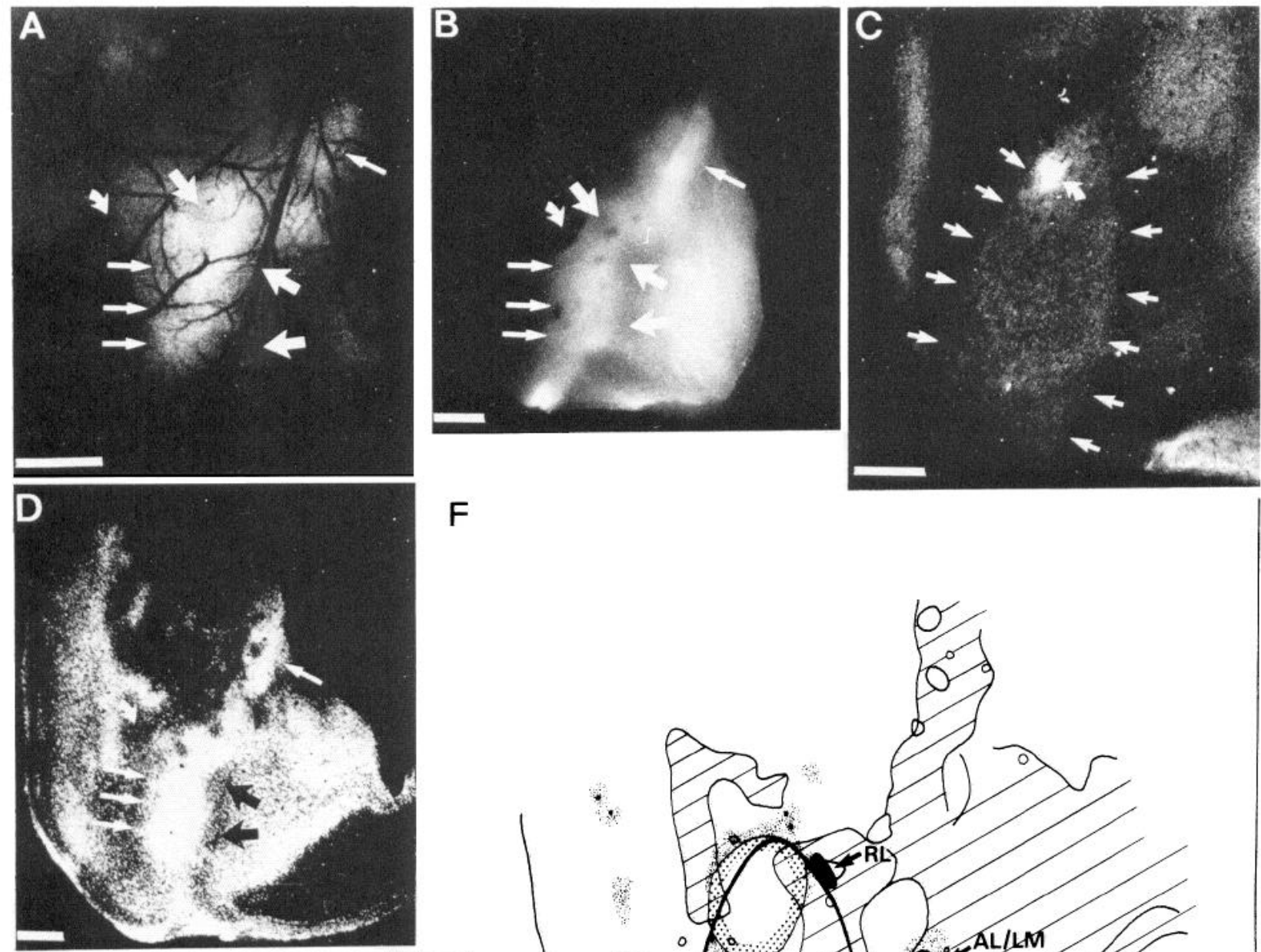

$\mathrm{F}$
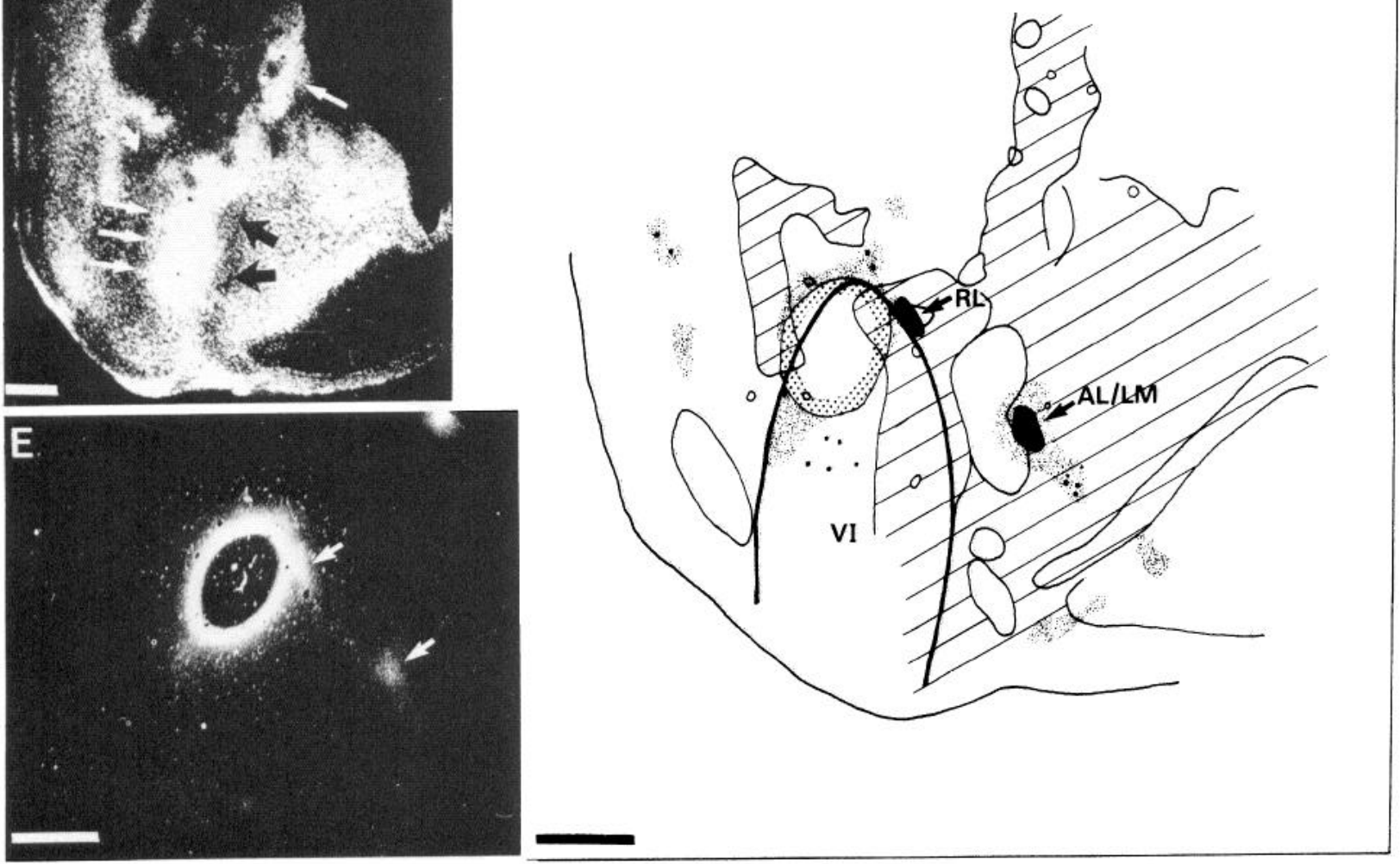

Figure 2. Vital tract tracing in rat cortex. A, Dorsolateral view of the right cortical hemisphere of a live, anesthetized rat, illuminated with UV light to reveal BB-labeled callosal connections. Arrows point to main features of the callosal pattern: the main callosal band (thin arrows at left), anterior occipital callosal ring (AOCR, top thick arrow), a callosal band on the medial border of area 3 (thin arrow at top right) a-callosal regions corresponding to zones AL and LM ( 2 thick arrows, top and bottom right, respectively). Curved arrow indicates small bleeding associated with the injection site. In this and subsequent figures anterior is $u p$, lateral is to the right. All parts of this figure are from the same cortical hemisphere. $B$, Dorsolateral view of the right cortical hemisphere following perfusion and fixation. The brain was viewed whole under UV illumination to reveal the pattern of BB-labeled callosal connections. Arrows point to the same locations indicated in $A$. $C$, Bright-field, high-contrast photograph of an unstained $40 \mu \mathrm{m}$ section of the right cortical hemisphere which was flattened and cut tangentially to the surface (see Materials and Methods). This view offers clear demarcation of area 17 boundaries. Straight arrows point to the lateral and medial borders of area 17, while the curved arrow points to the WGA-HRP injection site. The injection was placed at the anterior tip of area 17 using the in vivo visualized callosal pattern as a guide. It should be noted that in several cases the location of area 17 was estimated based only on its relation to the callosal pattern. In these cases, the figures do not contain exact demarcation of area 17 borders (i.e., Figs. 3, 5-7). D, A neighboring section to the one shown in $C$ but in this case illuminated with UV light to reveal the BB-labeled callosal projections. Arrows point to the same sites indicated in $A$ and $B$; curved arrow indicates injection site. Scale bar, $1 \mathrm{~mm}$. $E$, Same section as $C$ but after the section was reacted for HRP and photographed using dark-field illumination to reveal WGA-HRP-labeled projections. Arrows point to the 2 main labeled patches situated laterally to the injection site. $F$, Summary drawing of results from this case, superposing the callosally connected zones (diagonal lines), the myeloarchitectonically defined borders of area 17 (thick line), and the WGA-HRP-labeled projections (shaded regions). Fine shading indicates weak labeling, and filled patches indicate more intense labeling. Large dots represent regions where clusters of retrogradely labeled cell bodies were observed. Arrows indicate main labeled patches and the estimated extrastriate zones associated with them. Scale bars, $1 \mathrm{~mm}$. 


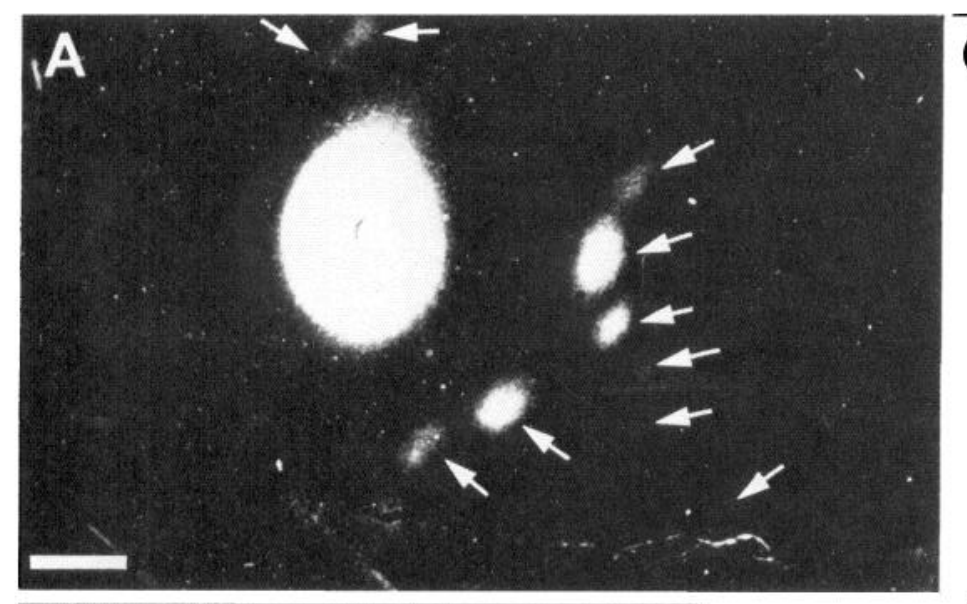

C
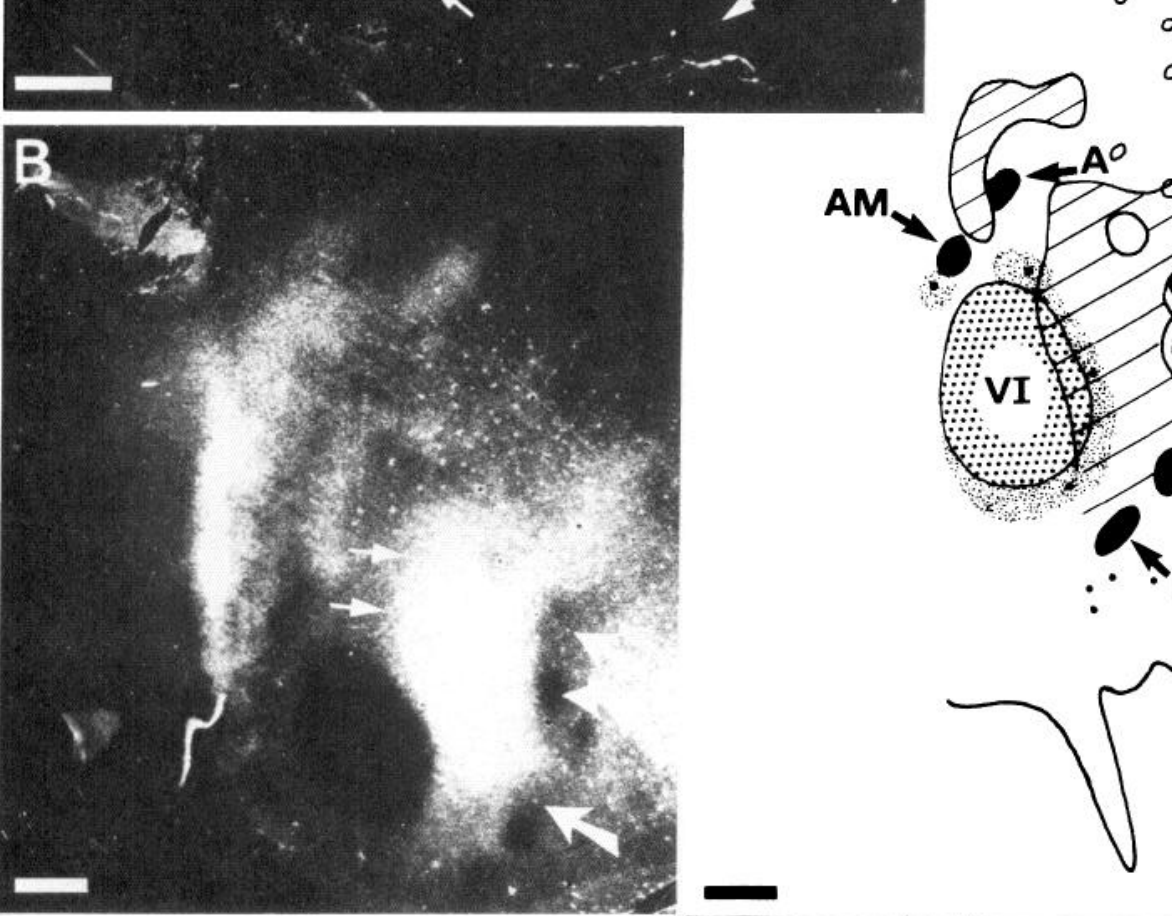

0

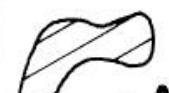

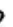

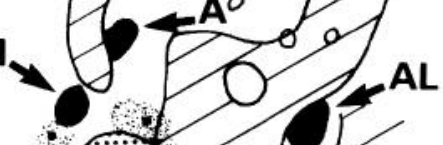
$\mathbf{A L}$
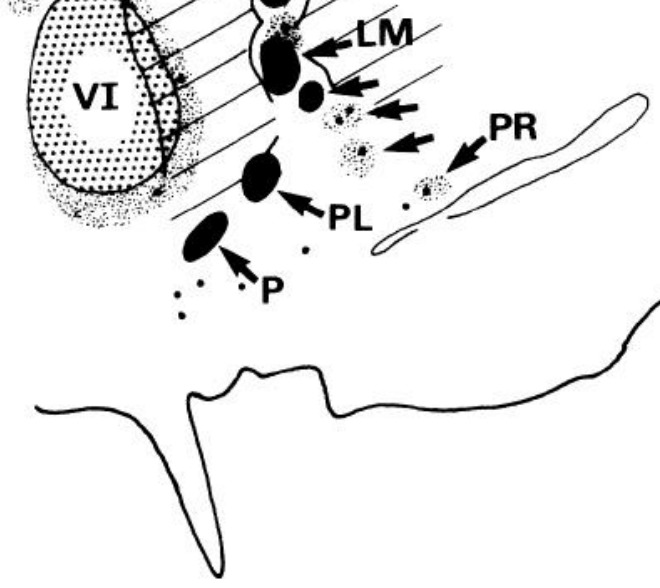

Figure 3. WGA-HRP injection into central area 17. $A$, Dark-field view of a tangential section from a flattened, right cortical hemisphere, reacted for HRP. Arrows point to locations of all labeled patches surrounding the injection site including those seen only at higher magnification. $B$, Same section as $A$, but viewed under UV illumination to reveal distribution of BB-labeled callosal connections. Arrows at left point to the main callosal band, while arrows at right point to 3 a-callosal zones, named, respectively, AL, LM, and PL. Scale bar, $1 \mathrm{~mm}$. $C$, Same case, drawing superposing the callosal pattern and the WGA-HRP-labeled connections. Note the correspondence between the patches and the a-callosal zones. Same symbols and conventions as in Figure 2. Scale bars, $1 \mathrm{~mm}$.

since their associated callosal rings were often difficult to discern in the BB labeling.

To illustrate the methodological strategy employed throughout this study, Figure 2 shows a case in which the anterior pole of area 17 was injected using the in vivo visualized callosal pattern for guidance. The sequence of experimental stages is illustrated in Figure 2, $A-F$. Figure $2 A$ shows the appearance of the in vivo visualized callosal pattern after the WGA-HRP injection was made. BB-labeled regions appear white underneath the dark epidural blood vessels. Straight arrows point to main callosal features which were useful in guiding tracer injections into the various cortical sites. These included the main callosal band encompassing the lateral border of area 17, several callosal rings within area $18 \mathrm{a}$, the prominent AOCR situated rostral to the main callosal band, and a rostrolateral band that defines the lateral border of somatosensory area 3 .

After brain perfusion and fixation the callosal pattern could be more easily discerned in the whole brain preparation due to clearing of the vasculature. This is illustrated in Figure $2 B$. The ability to view callosal connections at this stage was particularly useful for verifying location of the injection site relative to the callosal pattern in cases which were subsequently sectioned in the coronal or sagittal planes.

It has been demonstrated that the borders of brain nuclei can be revealed in unstained brain sections using appropriate illumination (Guzman-Flores et al., 1958). Adopting this approach in flattened cortex sections from middle layers revealed clear delineations of areas 17 and 41 and the barrel fields in area 3, particularly when viewed with dark-field illumination. Comparison of this "endogenous" pattern with myelin staining of the same section revealed that the 2 patterns were essentially identical. Consequently, we used this method in several cases to define the myeloarchitectonic borders of area 17. Figure $2 \mathrm{C}$ shows the clearly defined borders of area 17 as they appear in 


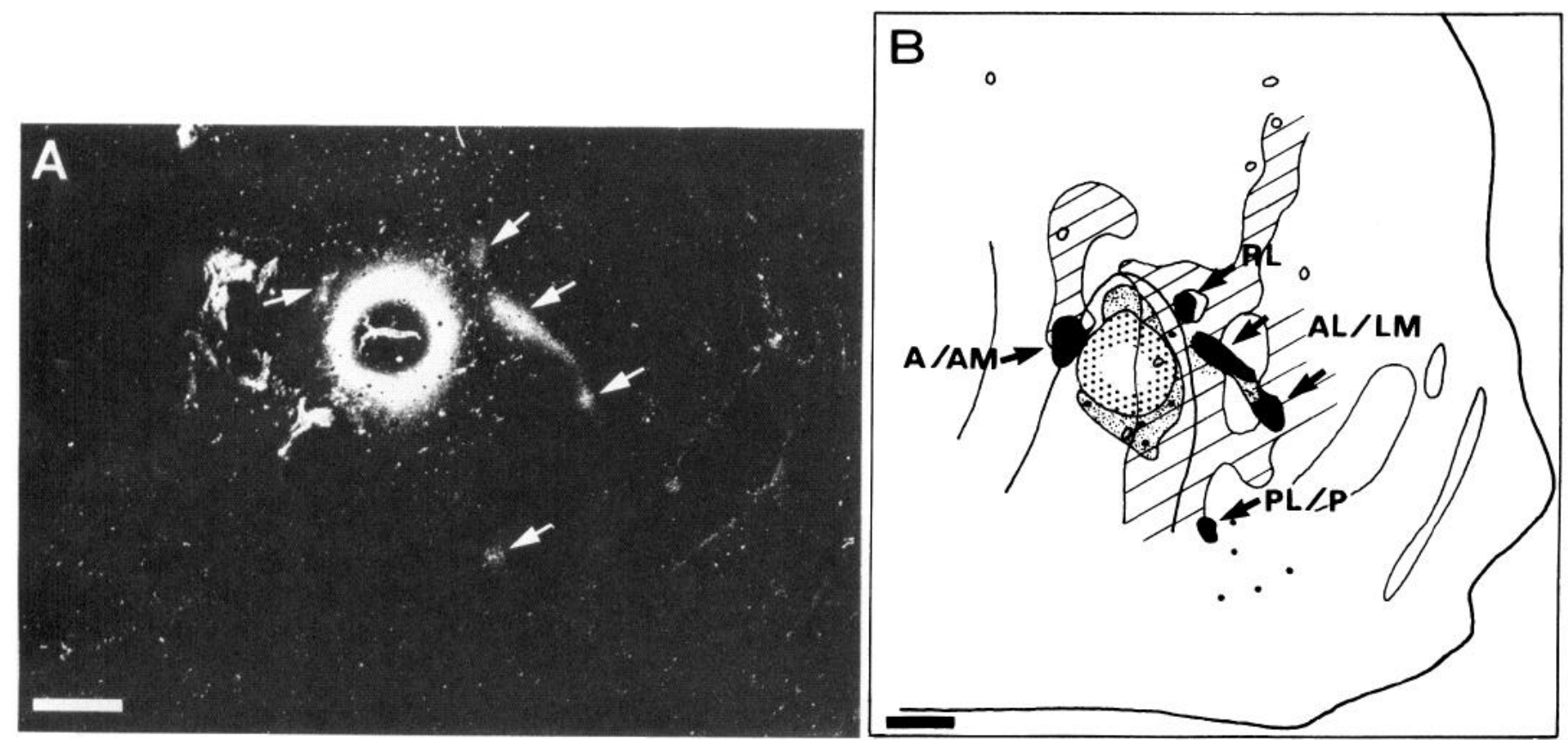

Figure 4. WGA-HRP injection into anterior area 17. A, Dark-field view of a tangential section reacted for HRP. Arrows point to main labeled patches surrounding the injection site. $B$, Graphic superposition of the callosal pattern and the WGA-HRP-labeled patches. Note higher incidence of labeling in rostral extrastriate cortex and in joint border zones. Same conventions as in Figure 2. Scale bars, $1 \mathrm{~mm}$.

an unstained, flattened cortical section of this case. Small arrows point to the borders of the oval shaped area 17. The curved arrow indicates location of the injection site close to the anterior tip of area 17.

The fact that it was possible to delineate the borders of area 17 without staining was advantageous because it allowed subsequent processing of the same sections for HRP. Illuminating these sections with UV light revealed that the HRP reaction did not degrade the BB labeling. Consequently, all 3 patterns-borders of area 17, the BB-labeled callosal connections, and the WGA-HRP-labeled ipsilateral corticocortical connectionscould be revealed in the same section. This provided enhanced precision in relating the 3 patterns to each other. In addition, neighboring sections were left unstained to show details of the callosal pattern around the injection site. Figure $2 D$ shows such a section from the same case, viewed under UV illumination. Arrows point to similar locations as in Figure 2, $A, B$. While there is great improvement in contrast and detail of the pattern compared with the whole-brain preparation, the main callosal features can be recognized in all 3 preparations. The same section shown in Figure $2 C$ was reacted for HRP, and the labeling pattern can be seen in Figure 2E. Arrows point to the two main labeled zones situated lateral and posterolateral to the injection site.

Finally, all 3 patterns were superposed using blood capillaries for alignment. Figure $2 F$ shows, schematically, the superposition of the callosal pattern, the myeloarchitectonic borders of area 17, and the WGA-HRP staining pattern. Note that this injection resulted in 2 clear patches situated in the rostral half of extrastriate cortex: one in the rostral zone RL and one roughly at the border between zones $\mathrm{AL}$ and $\mathrm{LM}$. At higher magnification, weaker, more posterior patches could also be discerned. It should be emphasized that in this and following figures, the graphic delineation of the HRP labeling pattern is based on inspection, at high magnification, of several sections throughout the depth of the cortex. Thus, information regarding the radial distribution of the label was "compressed." Two points should be noted. First, in essentially all cases, labeling was found both in cell bodies and in fine terminations, indicating the existence of reciprocal connections between patches. However, the relative density of labeled cell bodies and terminations varied markedly in different sections. Second, labeled patches were roughly aligned in the different sections, suggesting that they conformed to a vertical columnar organization.

The labeling pattern shown above, following injection in the anterior extreme of area 17, appeared markedly different from that reported following central area 17 injections (Olavarria and Montero, 1984). To verify that this discrepancy was due to the change in injection site and not to a difference in methodology, we repeated the central area 17 injection using the VTT approach. The results from this case are shown in Figure 3. Figure $3 A$ is a macrophotograph of the patterns of connections taken at low power. (Arrows point to main labeled zones including those seen only at higher power.) The injection resulted in a series of 10 discrete patches surrounding area 17 . Figure $3 B$ shows the BB-labeled callosal pattern in the same section. The main callosal band can be discerned, as well as the lateral a-callosal zones. The dark injection site can be seen situated medially to the main callosal band, within area 17 . When the 2 patterns were superposed, as shown in Figure $3 C$, the labeled patches overlapped the centers of the a-callosal zones on the medial and the lateral sides of area 17. While most of the extrastriate zones defined in Figure 1 contained labeled patches following the central injection in area 17, conspicuously missing was the most rostral pole of extrastriate cortex, which, as shown above, did receive projections from anterior striate cortex. These results are in good agreement with previous reports based on using ${ }^{3} \mathrm{H}-$ proline as the tracer (Olavarria and Montero, 1984).

The unexpected finding that rostral V1 selectively innervates the rostral aspects of extrastriate cortex was substantiated by 

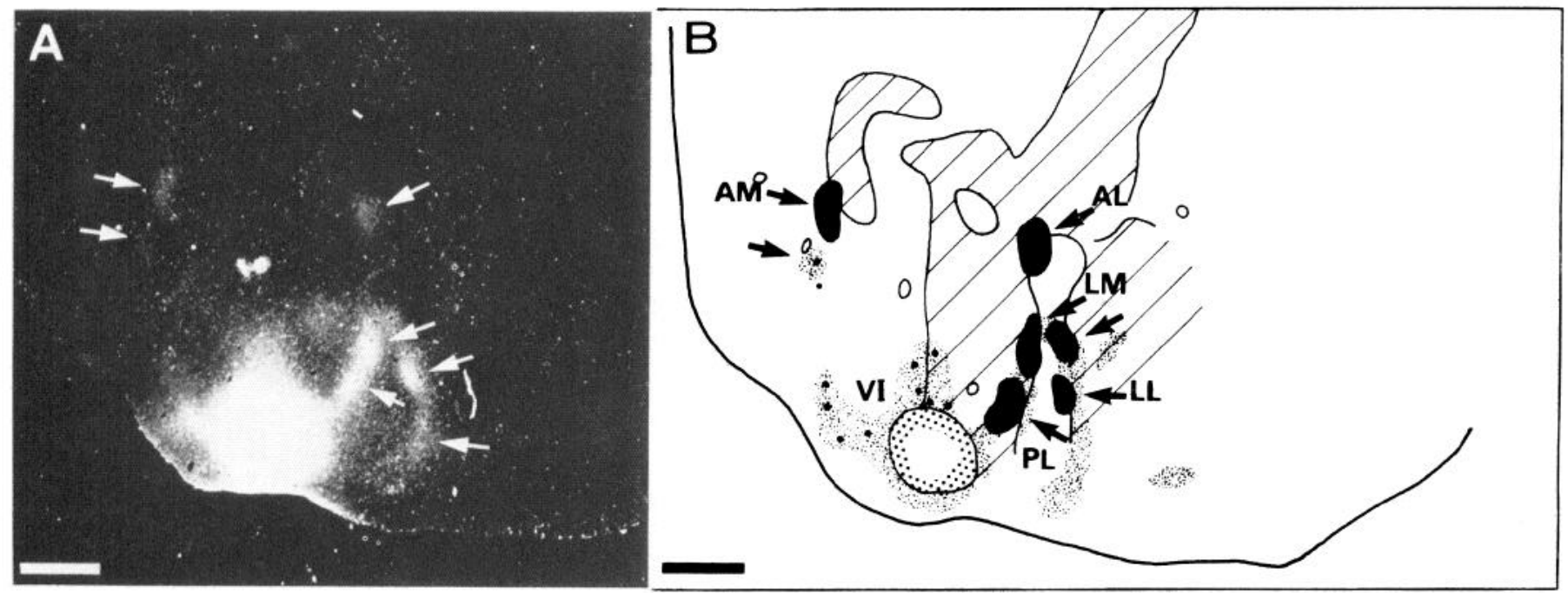

Figure 5. Caudal area 17 injections. A, Dark-field view of a tangential section reacted for HRP to reveal WGA-HRP-labeled patches in a case injected at caudolateral area 17. Arrows indicate location of main labeled patches. B, Graphic superposition of the callosal pattern and the WGAHRP-labeled patches. Note heavier concentration of labeling in caudal extrastriate cortex and its overlap with the "septa" of the calosally connected rings. Same conventions and symbols as Figure 2. Scale bars, $1 \mathrm{~mm}$.

injecting a site somewhat posterior to the one shown in Figure 2. The results are shown in Figure 4, with the HRP labeling shown in Figure $4 A$ and its relation to the callosal pattern and to area 17 borders in Figure $4 B$. Two points should be noted in this experiment. First, there was again clear labeling in rostral zone RL which was missing following the more central V1 injection. Second, most patches appeared to be confined to the joint borders of callosal rings and not to their centers. Thus, a labeled patch could be recognized at the border of zones A and $\mathrm{AM}$ on the medial side and along the AL/LM borders and possibly the PL/P borders on the lateral side. Note also that, in comparison with Figure 2, this case, which was injected slightly more caudally, showed increased labeling in caudal extrastriate cortex.

In order to complete the description of striate-extrastriate connections, we injected the extreme caudal parts of area 17. The results of a case injected at the caudolateral part of area 17 are shown in Figure 5. In this case, it was clear that most of the labeling now shifted to the more caudal aspects of extrastriate cortex. Note, however, that more locally, 2 additional changes
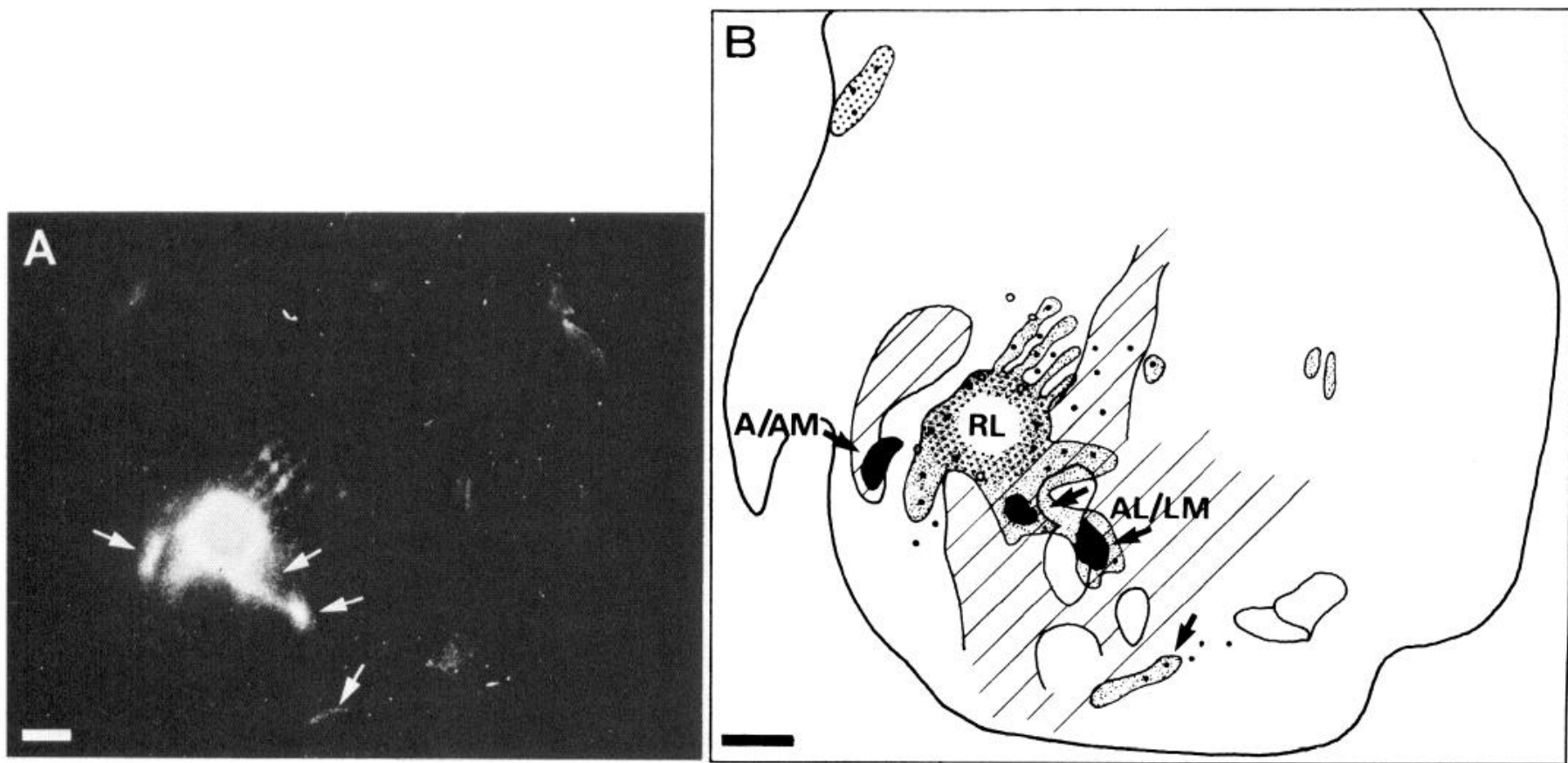

Figure 6. Rostrolateral extrastriate injection. A, Dark-field view of a tangential section reacted for HRP. Arrows point to main WGA-HRP-labeled patches. $B$, Graphic superposition of the callosal pattern with the WGA-HRP-labeled patches, indicating major labeling in the rostral half of extrastriate cortex and confined to joint-border zones. Same symbols as Figure 2. Scale bar, $1 \mathrm{~mm}$. 

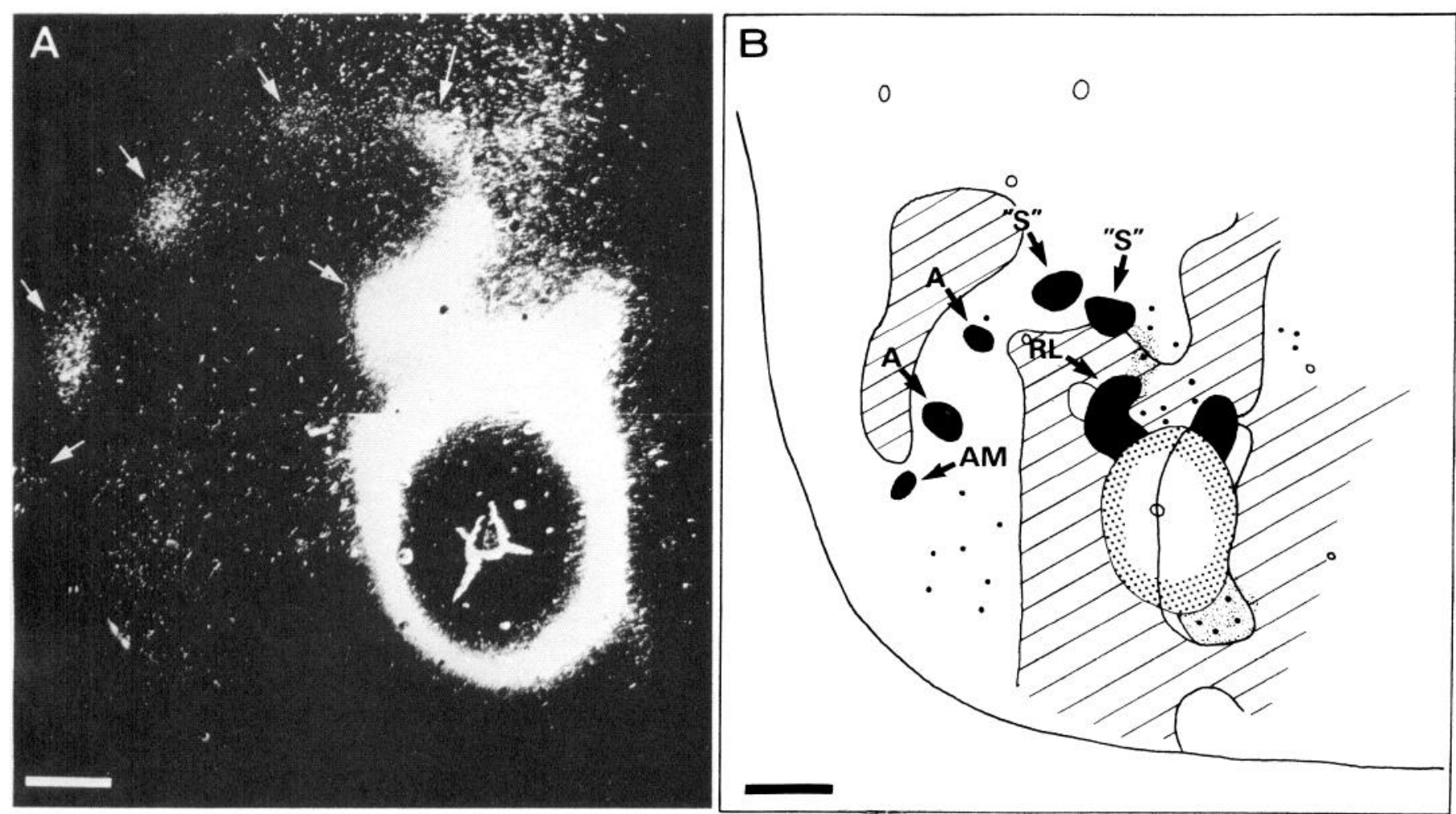

Figure 7. Large WGA-HRP injection overlapping parts of the anterolateral and lateromedial extrastriate zones. $A$, Dark-field view of a tangential section showing the "necklace" organization of WGA-HRP-labeled patches surrounding the anterior pole of area 17. Arrows point to main labeled patches. B, Superposition of the WGA-HRP-labeled patches and the callosal pattern. Note multiple patches within extrastriate zones A and "S." Same symbols and conventions as in Figure 2. Scale bars, $1 \mathrm{~mm}$.

can be recognized. First, as the injection site was displaced from rostral to caudal poles of area 17 , extrastriate labeling shifted away from the $\mathrm{A} / \mathrm{AM}$ and $\mathrm{AL} / \mathrm{LM}$ border zones. Second, as the injection site was displaced from the a-callosal body of area 17 (e.g., Fig. 3) to the callosally connected main band, there was a corresponding shift in extrastriate labeling from the a-callosal hollows to the callosally connected "septa" of the rings. These trends suggest that projections from medial and lateral area 17, as well as projections from rostral and caudal area 17 , tended to avoid each other.

In summary, the results from the striate cortex injections indicate that connections between striate and extrastriate cortices were widespread. However, these connections were not uniformly distributed. They were located in discrete patches which were in fixed relation to the callosal rings. Shifting the injection site within area 17 appeared to cause a global shift in the "center of gravity" of the cluster of labeled patches, as well as local displacements of individual patches within the confines of each callosal ring.

\section{Intrinsic extrastriate connections}

In view of the connectivity patterns found between striate and extrastriate cortex, it was interesting to study how they relate to interconnections within extrastriate cortex. Figure 6 shows results from a case in which a large injection of tracer was placed at the rostral zone RL. Despite the extensive injection site, which also spread rostrolaterally to area 3 and labeled the septa of barrel fields, the labeling was mostly confined to rostral extrastriate cortex. The overall pattern was similar to that found following injections at the rostral pole of area 17, with labeled patches mostly localized at specific, joint border zones (cf. Fig. 4).

To illustrate the full complexity of rostral connections, a large lateral injection was made, which included major parts of zones $\mathrm{AL}$ and $\mathrm{LM}$. The resulting pattern contained 6 rostral patches (Fig. 7A, arrows) organized along a crescent-shaped band. Interestingly, this band spanned smoothly from area $18 \mathrm{a}$ to $18 \mathrm{~b}$, with no apparent relation to their borders. On the other hand, it is clear that the cortical parcelation scheme adopted here was insufficient to capture the full complexity of the labeling pattern since more than one patch of label could be associated with some extrastriate subdivisions (i.e., zones A and "S" which each contained a pair of widely separated patches).

As the injection site was shifted caudally, the labeling pattern changed markedly. This is illustrated in Figure 8, which shows a case injected at the more caudal site in the center of area LM. Note that along with the shift in injection site, labeling in the extreme rostral zones was reduced, while more central parts of extrastriate cortex received increased projections.

Finally, Figure 9 shows the labeling pattern in the most caudally injected case from this series, which received a large injection overlapping the posterior zones PL and possibly P. Here, the rostral pole of extrastriate cortex was essentially devoid of label. This labeling pattern was quite similar to the one observed following caudal area 17 injections (cf. Fig. 5).

Injections medial to area 17 , in area $18 \mathrm{~b}$, gave results which followed similar trends to those seen in area 18a injections. Figure 10 illustrates this point by comparing the labeling fol- 

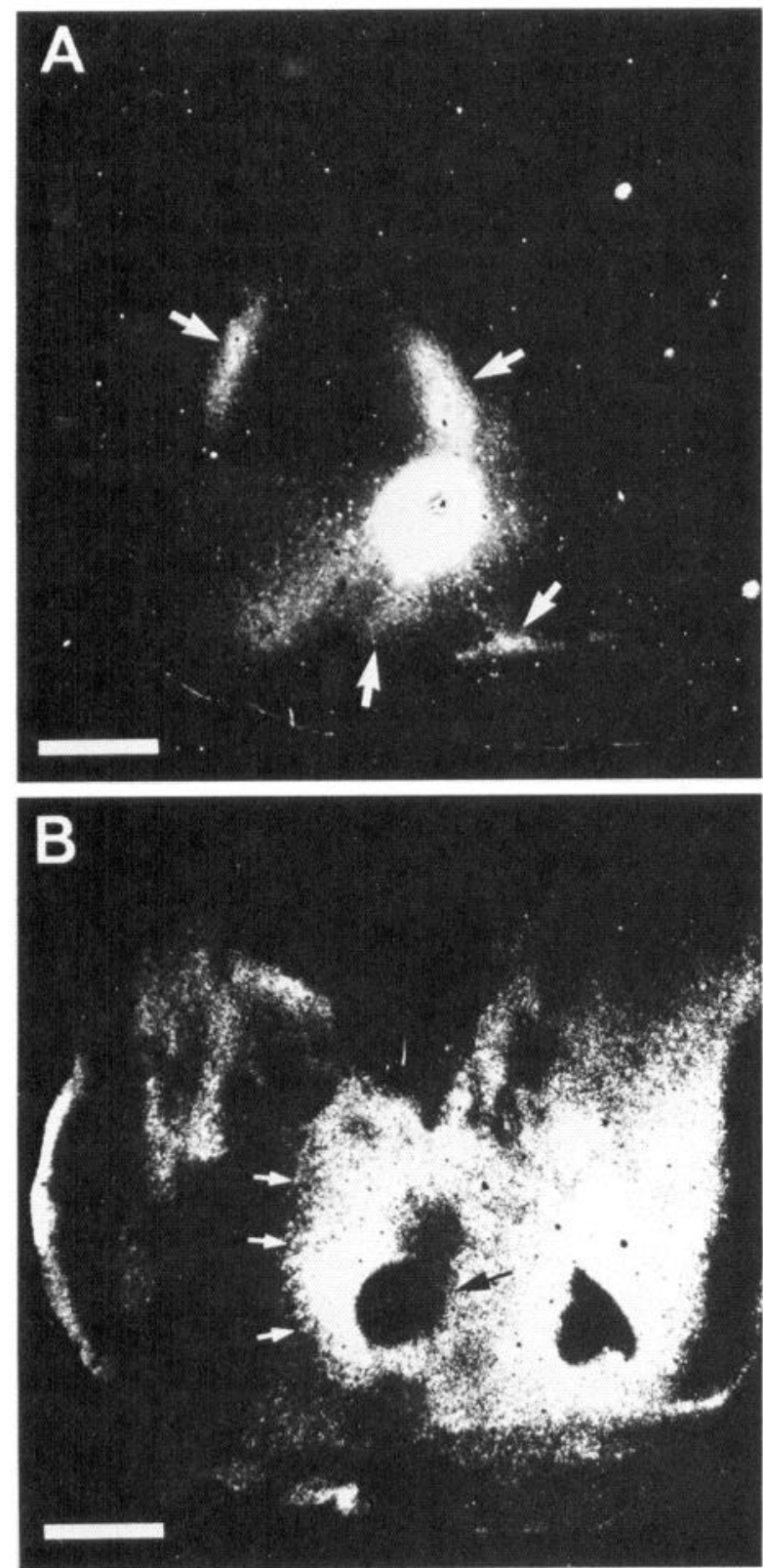
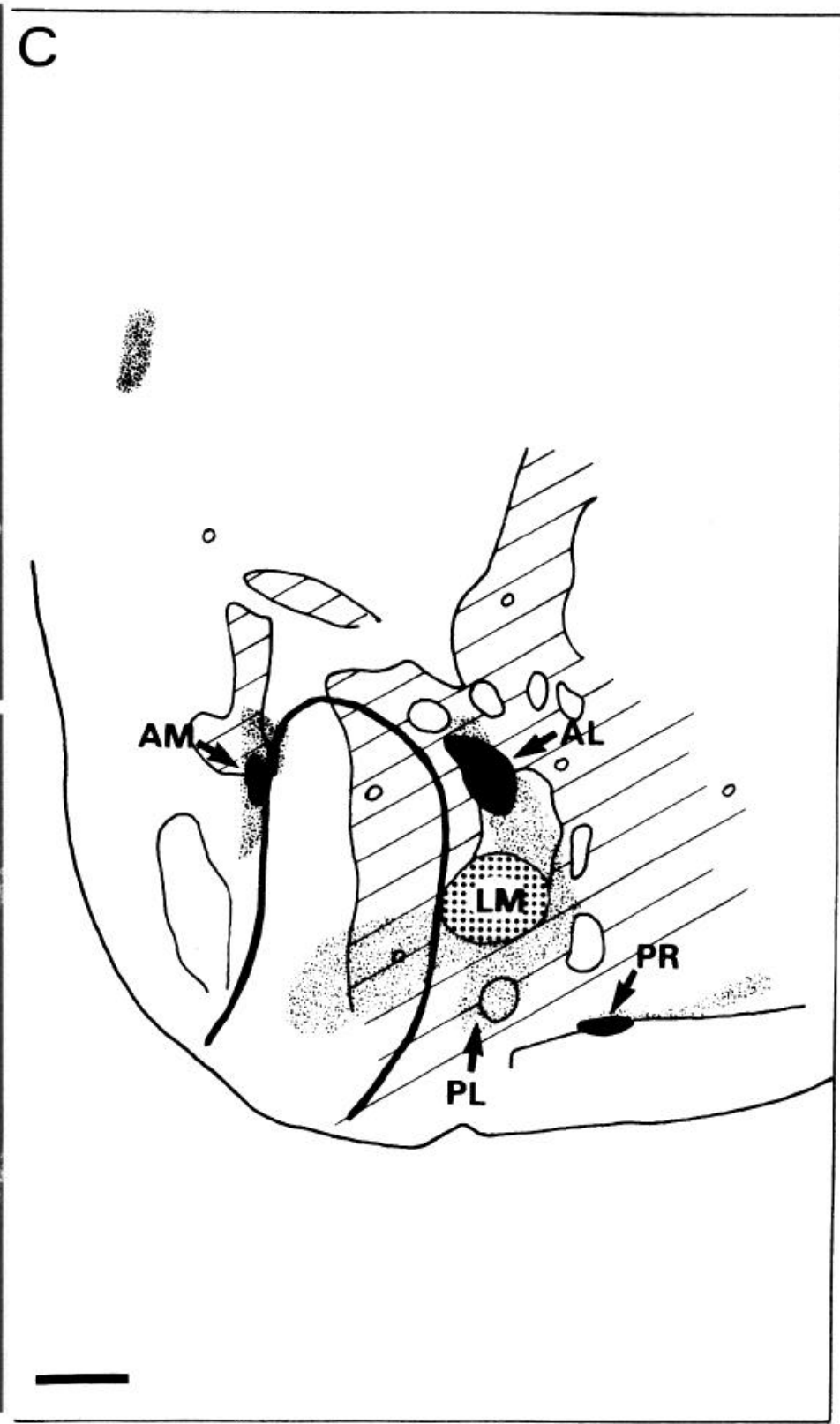

Figure 8. WGA-HRP injection in the lateromedial extrastriate zone. $A$, Dark-field view of a tangential section showing pattern of WGA-HRP labeling. Arrows point to main labeled patches. B, Same section illuminated with UV to reveal the callosal pattern. White arrows point to the main callosal band, while black arrow points to the injection site lateral to it. $C$, Graphic superposition of the WGA-HRP-labeled patches, the callosal pattern, and the myeloarchitectonically defined borders of area 17. Rostral extrastriate labeling was reduced as the injection site moved caudally. Same symbols and conventions as in Figure 2. Scale bars, $1 \mathrm{~mm}$.

lowing a rostral injection (zone A, Fig. 10, $A, B$ ) with a caudal injection (zone M, Fig. 10, $C, D$ ). The results showed extensive interconnections between areas $18 \mathrm{~b}$ and $18 \mathrm{a}$, which were arranged in multiple patches. However, as the injection site shifted from rostral $18 \mathrm{~b}$ to caudal $18 \mathrm{~b}$, there was a tendency for more caudal patches to appear and rostral patches to disappear. Note that here again, projections from callosal (Fig. 10, $A, B$ ) and a-callosal (Fig. 10, $C, D$ ) zones appeared to avoid each other.

\section{Discussion}

The results from all the cases described in the present study demonstrate that striate and extrastriate cortical sites have extensive and widespread interconnections which are organized in multiple, discrete patches. Closer examination of these projections reveals consistent relationships to the callosal pattern and to the myeloarchitectonic borders of area 17. In comparing the different projection patterns, certain organizational trends can be recognized. First, cortical sites tend to be more extensively connected to nearby targets than to distant ones. Thus, rostral and caudal injections in striate cortex emphasized labeling in rostral and caudal extrastriate cortex, respectively (e.g., compare Figs. 4 and 5). Within extrastriate cortex, the same trend is manifested in that rostral and caudal extrastriate injections tend to label their neighboring patches more extensively than distant ones (e.g., compare Figs. 6 and 9). In that respect, it is interesting to note that Miller and Vogt (1984) have reported 

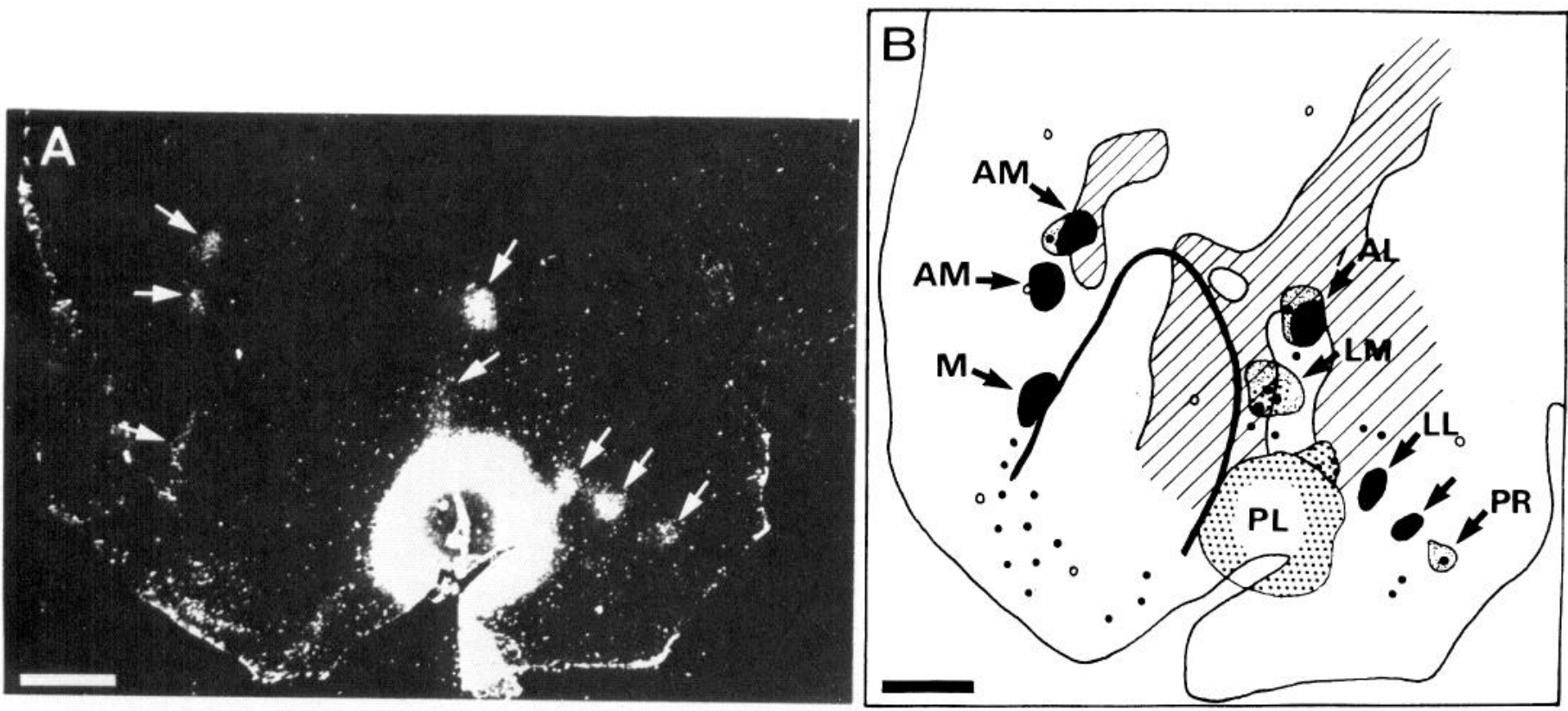

Figure 9. WGA-HRP injection in the posterolateral extrastriate zone. $A$, Dark-field view of a tangential section showing pattern of WGA-HRP labeling. Arrows point to main labeled patches. B, Graphic superposition of callosal pattern, WGA-HRP labeling and the myeloarchitectonically defined borders of area 17. Note higher incidence of labeling in caudal extrastriate cortex and its absence rostrally. Same symbols and conventions as in Figure 2. Scale bars, $1 \mathrm{~mm}$.

a similar trend for the medial and lateral sides of striate cortex, whose projections emphasized the corresponding medial (area $18 \mathrm{~b})$ and lateral (area 18a) extrastriate targets.

It should be noted that high-magnification viewing of the labeled patches revealed that the level of tracer transport in individual neurons was similar for nearby and distant targets. Only the number of labeled neurons and the size of projections tended to vary with target distance.

When comparing the different experiments of this study, using the callosal pattern as a fixed reference, a second trend becomes apparent: extrastriate sites receiving inputs from common cortical loci tend to be interconnected. Thus, zone RL and the borders of zones $\mathrm{AL} / \mathrm{LM}$ and $\mathrm{A} / \mathrm{AM}$, all receiving inputs from rostral striate cortex, were also mutually interconnected (e.g., compare Figs. 4 and 6). Similarly, sites receiving inputs from caudal striate cortex tended to be interconnected (compare Figs. 5 and 9). Compatible with this trend is also the tendency for callosally connected zones to interconnect with each other (Figs. 5; 10, A, B).

Finally, projections from rostral and caudal striate cortex, as well as projections from medial and lateral striate cortex, tended to avoid each other, resulting in patterns of interdigitation at regions of overlap (cf. Figs. 4 and 5 as examples of rostrocaudal interdigitation, and Figs. 5 and 3 for mediolateral interdigitation). This trend supports a similar suggestion for mirror-symmetric interdigitation put forward by Olavarria and Montero (1984) based on their analysis of central area 17 projections.

In summary, the results suggest that extrastriate cortex organization follows 3 trends: (1) Neighboring connections are more extensive; (2) sites receiving common input are directly interconnected; (3) projections from opposite poles in striate cortex interdigitate at regions of overlap. To schematically illustrate these trends Figure 11 shows the projection patterns from one rostral and one caudal extrastriate site. In this scheme, regions receiving connections from rostral and caudal area 17 are indicated, respectively, by dark and light shading. Note that darkly shaded (rostrally connected) zones are more extensive in rostral extrastriate cortex, while lightly shaded (caudally connected) zones are localized more caudally. All sites receiving common inputs from one pole of striate cortex are mutually interconnected and tend to interdigitate.

\section{Relationship to visuotopic maps}

An important question is how do the multiple patches of connections revealed in rat extrastriate cortex relate to the known electrophysiological maps of this territory? Comparing the topography of connections found here with published visuotopic maps (Montero et al., 1973a; Espinoza and Thomas, 1983; Thomas and Espinoza, 1987) suggests that in several instances the 2 are correlated, as for example, with regard to the location of lower visual field representations: According to physiological mapping studies, they are located at the rostral part of striate cortex, at the RL region and at what appears to be the AL/LM border zone within extrastriate cortex. The present study shows that these regions tended to be interconnected. Such correlations could imply that in rat visual cortex, all sites which represent similar points in visual space are also interconnected. However, more direct, within-animal comparisons of retinotopic mapping and connections will be needed to firmly establish this point.

\section{The issue of multiplicity of visual areas in rat extrastriate} cortex

Should the belt of tissue adjoining striate cortex be considered a single area or several visual areas? The results suggest that this territory has an intermediate organization which does not fall clearly into either category. To illustrate this point, it is useful to compare the present results with patterns of corticocortical connections in cases which have better defined organization. First, the trend for preferential projections to nearby sites is characteristic of single, global map organization. This 


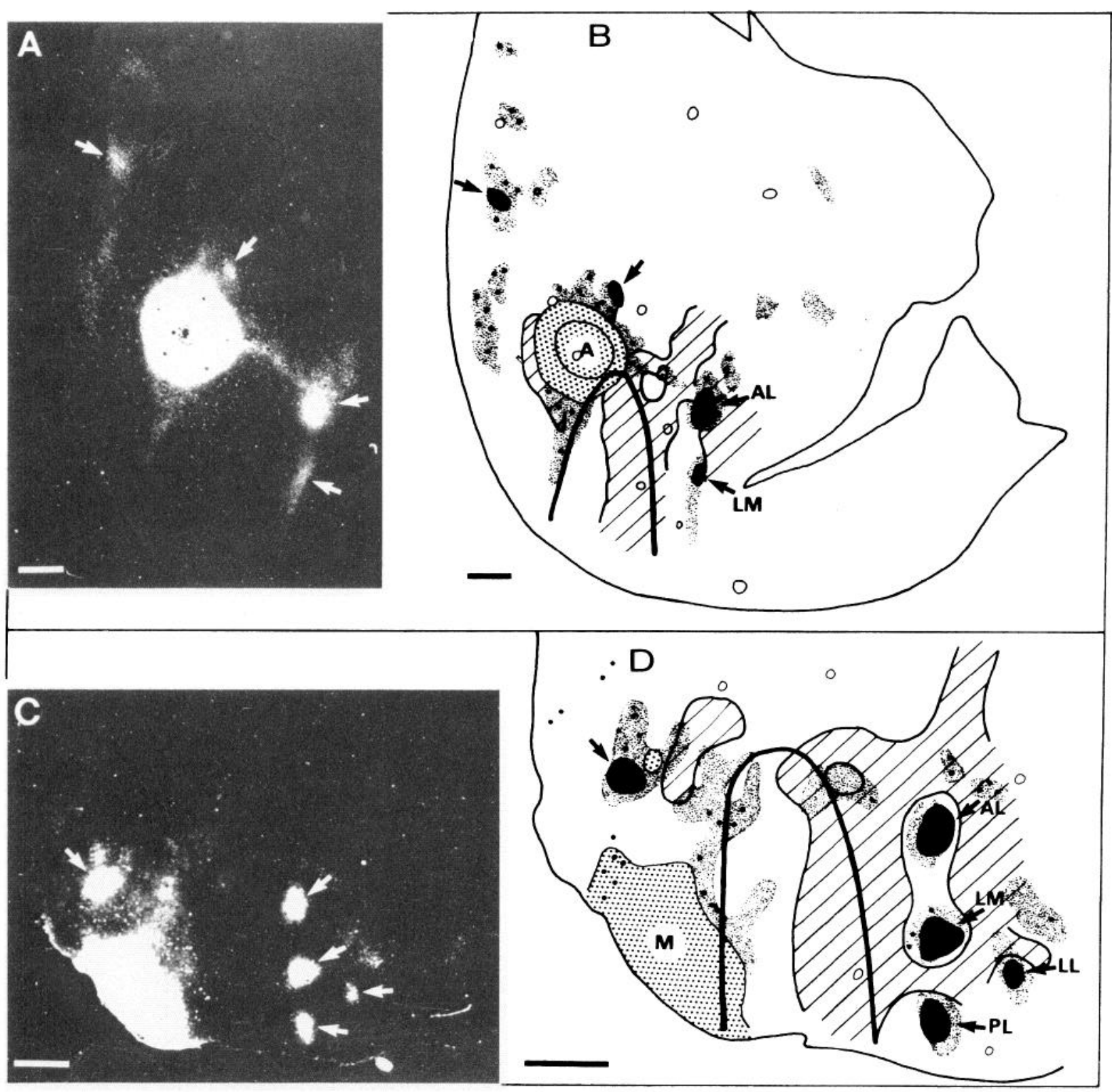

Figure 10. WGA-HRP injections in the posterior and anterior poles of area 18b. $A$, Dark-field photograph of a tangential, HRP-tested section in a case which received WGA-HRP injection into zone anterior, at the anterior pole of area $18 \mathrm{~b}$. Arrows point to main labeled patches in visual cortex. $B$, Graphic superposition of the callosal pattern, WGA-HRP labeling and the myeloarchitectonically defined borders of area 17. Labeled patches can be found in the rostral zones and overlap with the "septa" of callosal rings. $C$, Same procedure as in $A$ but in a case which received WGA-HRP injection into zone medial at the caudal pole of area $18 \mathrm{~b}$. Arrows point to main labeled patches. $D$, Graphic superposition of the callosal pattern, WGA-HRP labeling and the myeloarchitectonically defined borders of area 17 . Labeled patches were located more caudally and overlapped the a-callosal hollows within the callosal rings. Same symbols and conventions as in Figure 2. Scale bars, $1 \mathrm{~mm}$.

trend can be recognized in connections of classically defined single areas such as the intrinsic connections within cat striate cortex (Gilbert and Wiesel, 1983). More importantly, a similar trend can be recognized in connections of area $\mathrm{V} 2$ of cat and monkey, also considered a single visual area. Thus, in cat (Montero, 1981) and monkey (Wong-Riley, 1979; Livingstone and Hubel, 1987), as well as the tree shrew (Sesma et al., 1984), single striate cortex injections result in a cluster of labeled patches in V2. As the injection site is shifted in striate cortex, there is a corresponding shift in the location of the labeled patches in area $\mathrm{V} 2$.

In other aspects as well, there are similarities between rat extrastriate cortex and area V2 of cat and monkey. The visual map in both cases is irregular: it contains, in the cat, visuotopic "islands" which also correspond to callosal bands running across the width of area 18 (Tusa et al., 1979; Sanides and Albus, 1980). These bands resemble the callosal rings in area 18a of the rat. Similarly, in monkey V2, the compartmental parcelation is well established both on connectional and histochemical grounds (e.g., DeYoe and Van Essen, 1985; Livingstone and Hubel, 1987). Finally, intrinsic connections in area 18 of cat and monkey are extensive and organized in a patchy manner (Rockland, 1985; Matsubara et al., 1987; Levay, 1988), again resembling the intrinsic connections within rat extrastriate cor- 


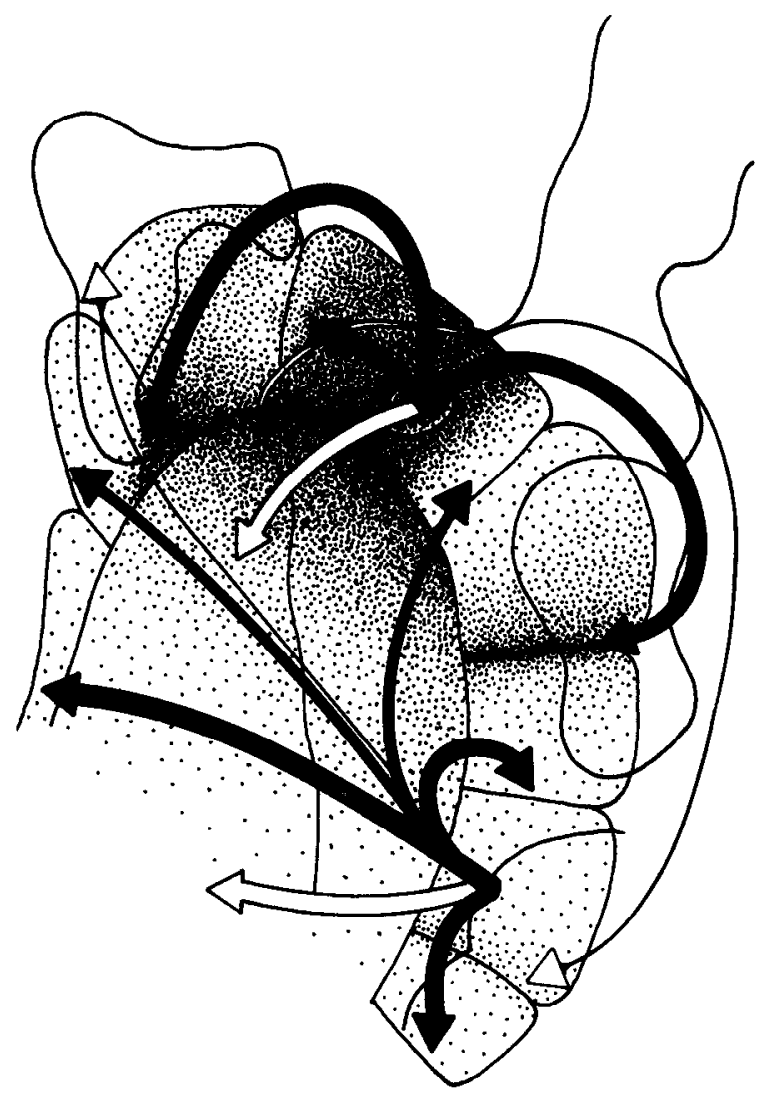

Figure 11. Suggested scheme for the organization of visual corticocortical connections in the rat. Only zones abutting area 17 are shown. The borders of area 17,9 extrastriate zones, and the callosal pattern are drawn in thick lines. Different shadings indicate associations with different parts of striate cortex. Rostral and caudal parts are indicated by lighter and darker shading, respectively. Arrows indicate zones that are reciprocally connected. Wider arrows represent more extensive interconnections; filled arrows indicate connections within extrastriate cortex, while unfilled arrows indicate striate-extrastriate connectivity. For clarity, only anterior zone RL and posterior zone PL connections are shown. Note that zones connected to similar points in striate cortex are interconnected, that these connections are interdigitated, and that rostral and caudal parts of extrastriate cortex tend to be associated with rostral and caudal striate cortex, respectively. This association imposes a global rostrocaudal axis on extrastriate cortex.

tex described here. Thus, various organizational similarities make it plausible that the band of tissue adjoining striate cortex in the rat is a global map which comprises the rat homolog of cat and monkey area $\mathrm{V} 2$.

However, in contrast to the cat and monkey, in which the patchy labeling caused by single injections is confined to a relatively small part of the entire cortical map, the multiplicity of labeling in rat cortex is far more extensive and occupies most of the global map. Furthermore, the patterns of interdigitation, as well as the multiple representations revealed in electrophysiological mapping experiments, suggest a consistent and farreaching fragmentation of the global map. The scheme that is suggested for rat extrastriate cortex is thus a dual one: while the belt adjoining striate cortex consists of a single, global map, this map is exceedingly fragmented and contains multiple visuotopic representations throughout its entire extent.

In view of such complex architecture, the issue of what constitutes a separate area is heavily dependent on the criteria used. Again, this is not a unique situation for the rat. In cat cortex, physiological mapping criteria have parceled the territory adjoining the lateral suprasylvian sulcus into 6 areas (Palmer et al., 1978), while, on the basis of connectional similarities, Sherk (1986) has included most of this territory in a single area. On the other hand, relying on connectional and functional arguments, Burkhalter et al. (1986) have divided the band of tissue adjoining area V2 in the macaque into 2 areas: V3 and VP, whereas based on visuotopic mapping criteria these subdivisions were incorporated in a single area (e.g., Gattas et al., 1988).

The complex architecture of rat visual cortex suggested by this study illustrates that in some instances the dichotomous parcelation of a cortical territory into either single or multiple areas might be forced. Thus, such cases further indicate that between these 2 extremes there may exist a spectrum of intermediate organizational patterns having different levels of fragmentation of the visual map.

\section{References}

Barlow, H. B. (1986) Why have multiple cortical areas? Vision Res. 26: $81-90$.

Burkhalter, A., D. J. Felleman, W. T. Newsome, and D. C. Van Essen (1986) Anatomical and physiological asymmetries related to visual areas V3 and VP in the macaque extrastriate cortex. Vision Res. 26: 63-80.

Caviness, V. S., Jr. (1975) Architectonic map of neocortex of the normal mouse. J. Comp. Neurol. 164: 247-264.

Choudhury, B. P. (1978) Retinotopic organization of the guinea pig's visual cortex. Brain Res. 144: 19-20.

Cusick, C. G., and R. D. Lund (1981) The distribution of the callosal projection to the occipital visual cortex in rats and mice. Brain Res. 214: 239-259.

Desimone, R., S. J. Schein, J. Morgan, and L. Ungerleider (1985) Contour, color and shape analysis beyond the striate cortex. Vision Res. 25: 441-452.

DeYoe, E. A., and D. C. Van Essen (1985) Segregation of efferent connections and receptive field properties in visual area V2 of the macaque. Nature 317: 58-61.

Drager, U. C. (1975) Receptive fields of single cells and topography in mouse visual cortex. J. Comp. Neurol. 160: 269-290.

Espinoza, S. G., and H. C. Thomas (1983) Retinotopic organization of striate and extrastriate visual cortex in the hooded rat. Brain Res. 272: 137-144.

Gattas, R., A. P. B. Sousa, and C. G. Gross (1988) Visuotopic organization and extent of $\mathrm{V} 3$ and $\mathrm{V} 4$ of the macaque. J. Neurosci. 8 : 1831-1845.

Gilbert, D. D., and T. N. Wiesel (1983) Clustered intrinsic connections in cat visual cortex. J. Neurosci. 3: 1116-1133.

Guzman-Flores, C., M. Alvaraz, and A. Fernandez-Guardiola (1958) Rapid procedure to localize electrodes in experimental neurophysiology. Bol. Estud. Med. Biol. 16: 29-31.

Hall, W. C., J. H. Kaas, H. Killackey, and I. T. Diamond (1971) Cortical visual areas in the grey squirrel (Sciurus carolinensis): A correlation between cortical evoked potential maps and architectonic subdivisions. J. Neurophysiol. 34: 437-457.

Illing, R. B., and H. Wassle (1979) Visualization of the HRP reaction product using the polarization microscope. Neurosci. Lett. 13: 7-11.

Krieg, W. J. S. (1946) Connections of the cerebral cortex I. The albino rat. A. Topography of the cortical areas. J. Comp. Neurol. 84: 221275.

Levay, S. (1988) Patchy intrinsic projections in visual cortex, area 18, of the cat: Morphological and immunocytochemical evidence for an excitatory function. J. Comp. Neurol. 269: 265-274.

Livingstone, M. S., and D. H. Hubel (1987) Connections between layer $4 \mathrm{~B}$ of area 17 and thick cytochrome oxidase stripes of area 18 in the squirrel monkey. J. Neurosci. 7: 3371-3377.

Malach, R. (1987) In vivo visualization of callosal pathways: A novel approach to the study of visual cortex organization. Soc. Neurosci. Abstr. 13: 5.12.

Malach, R. (1988a) Connectivity patterns within rat extrastriate cortex revealed with the aid of vital tracing of callosal connections. Soc. Neurosci. Abstr. 14: 451.7. 
Malach, R. (1988b) In vivo visualization of callosal connections: A novel approach to the study of cortical organization. J. Neurosci. Methods 25: 225-238.

Malach, R., and A. M. Graybiel (1986) Mosaic architecture of the somatic sensory-recipient sector of the cat's striatum. J. Neurosci. 6: 3436-3458.

Matsubara, J. A., M. S. Cynader, and N. V. Swindale (1987) Anatomical properties and physiological correlates of the intrinsic connections in cat area 18. J. Neurosci. 7: 1428-1446.

Mesulam, M. M. (1978) Tetramethyl benzidine for horseradish peroxidase neurohistochemistry: A non-carcinogenic blue reaction product with superior sensitivity for visualizing neural afferents and efferents. J. Histochem. Cytochem. 25: 106-117.

Miller, M. W., and B. A. Vogt (1984) Direct connections of rat visual cortex with sensory, motor, and association cortices. J. Comp. Neurol. 226: 184-202.

Montero, V. M. (1981) Topography of the cortico-cortical connections from the striate cortex in the cat. Brain Behav. Evol. 18: 194-218.

Montero, V. M., A. Rojas, and F. Torrealba (1973a) Retinotopic organization of striate and peristriate visual cortex in the albino rat. Brain Res. 53: 197-201.

Montero, V. M., H. Bravo, and V. Fernandez (1973b) Striate-peristriate cortico-cortical conncctions in the albino and gray rat. Brain Res. 53: 202-207.

Olavarria, J., and V. M., Montero (1981) Reciprocal connections between the striate and extrastriate cortical visual areas in the rat. Brain Res. 217: 358-363.

Olavarria, J., and V. M. Montero (1984) Relation of callosal and striate-extrastriate cortical connections in the rat: Morphological definition of extrastriate visual areas. Exp. Brain Res. 54: 240-252.

Palmer, L. A., A. C. Rosenquist, and R. J. Tusa (1978) The retinotopic organization of the lateral suprasylvian areas in the cat. J. Comp. Neurol. 177: 237-256.

Rockland, K. S. (1985) A reticular pattern of intrinsic connections in primate area V2 (area 18). J. Comp. Neurol. 235: 467-478.
Sanides, D., and K. Albus (1980) The distribution of interhemispheric projections in area 18 of the cat: Coincidence with discontinuities of the representation of the visual field in the second visual area (V2). Exp. Brain Res. 38: 237-240.

Sesma, M. A., V. A. Casagrande, and J. H. Kaas (1984) Cortical connections of area 17 in tree shrews. J. Comp. Neurol. 230: 337351

Sherk, H. (1986) Location and connections of visual cortical areas in the cat's suprasylvian sulcus. J. Comp. Neurol. 247: 1-31.

Thomas, H. C., and S. G. Espinoza (1987) Relationships between interhemispheric cortical connections and visual areas in hooded rats. Brain Res. 417: 214-224.

Tiao, Y. C., and C. Blakemore (1976) Functional organization in the visual cortex of the golden hamster. J. Comp. Neurol. 68: 459-482.

Tusa, R. J., A. C. Rosenquist, and L. A. Palmer (1979) Retinotopic organization of areas 18 and 19 in the cat. J. Comp. Neurol. 185 : $657-678$.

Van Essen, D. C. (1985) Functional organization of primate visual cortex. In Cerebral Cortex, Vol. 3, A. Peters and E. G. Jones, eds., pp. 259-329, Plenum, New York.

Van Essen, D., and J. H. R. Maunsell (1983) Hierarchical organization and functional streams in the visual cortex. Trends Neurosci. 6: 370375.

Van Essen, D. C., W. T. Newsome, and J. L. Bixby (1982) The pattern of interhemispheric connections and its relationship to extrastriate visual areas in the macaque monkey. J. Neurosci. 2: 265-283.

Wagor, E., N. J. Mangini, and A. L. Pearlman (1980) Retinotopic organization of striate and extrastriate visual cortex in the mouse. J. Comp. Neurol. 193: 187-202.

Welker, C., and T. A. Woolsey (1974) Structure of layer IV in the somatosensory cortex of the rat: Description and comparison with the mouse. J. Comp. Neurol. 158: 437-454.

Wong-Riley, M. T. T. (1979) Columnar cortico-cortical interconnections within the visual system of the squirrel and macaque monkeys. Brain Res. 62: 201-217. 